\title{
Standbunkt
}

Feministische Perspektiven in der Wirtschaftsforschung

\section{Die Krise der reproduktiven Arbeit}

\author{
Feministische Perspektiven auf Nachhaltigkeit? Beim Begriff \\ Feminismus denken viele ausschließlich an die rechtliche \\ Gleichstellung von Frauen. Damit würde sich zumindest \\ für Deutschland eine feministische Perspektive erübrigen. \\ In welcher Hinsicht geht die feministische Forschung \\ darüber hinaus? Von Tanja von Egan-Krieger
}

Z unächst wird unter Geschlechtergerechtigkeit beispielsweise auch der gleiche Zugang zu und die gleiche Teilhabe an Erwerbsarbeit und Einkommen, Macht, Gesundheitsvorsorge und Bildung verstanden. Daran anschließend wurde eine Reihe an Ungerechtigkeiten aufgedeckt. In den Ländern des Südens tragen Frauen etwa die Hauptlast der gesellschaftlich notwendigen Arbeit und sind am stärksten von den Umweltzerstörungen betroffen.

Eine weitere wesentliche Stoßrichtung der feministischen Forschung ist die Untersuchung von Grundannahmen in Theorien, vor allem hierarchischer Dichotomien, deren unterschiedliche Bewertung in der Theorie auf der Kategorie des sozialen Geschlechts beruht. Eine dieser Dichotomien, die insbesondere für den Nachhaltigkeitsdiskurs relevant ist, ist die Kategorie der (Re)Produktivität. Nach Biesecker und Hofmeister wurden die ökologische sowie die sozial weibliche Produktivität (soziale $\mathrm{Zu}$ weisungen an die Geschlechter) als „Reproduktivität“ aus der Ökonomik ausgegrenzt.

\section{Ausgrenzung weiblicher Leistungen}

Diese Ausgrenzung der sozial weiblichen und der ökologischen Leistungen aus dem Gegenstandsbereich der ökonomischen Theorie habe die industrielle Praxis begünstigt, denn ihr korrespondiert eine geringe Wertschätzung der sozial weiblichen und der ökologischen
Leistungen. Die Krise der „reproduktiven" Arbeit (Zerstörung von Familienleben, „Überalterung“ der Gesellschaft und vieles mehr) und die ökologische Krise seien in diesem Sinne gleichursprünglich. Damit kann die Kategorie der (Re)Produktivität eine Brückenfunktion zwischen der sozialen und der ökologischen Dimension von Nachhaltigkeit einnehmen.

Die Produktivität der sogenannten "Reproduktionsarbeit" wird inzwischen zwar weitgehend erkannt und der Arbeitsbegriff in der Folge um sorgende Tätigkeiten und bürgerschaftliches Engagement erweitert, aber dies rein additiv. Stattdessen müsste der Begriff Arbeit neu konzipiert werden. Was genau verstehen wir unter Arbeit? Welche Bedeutung schreiben wir den als Arbeit bezeichneten Tätigkeiten damit zu? Aus welchen Gründen tun wir dies?

\section{Irreführende Metaphern}

Die Produktivität von Natur wird vor allem in der Ökologischen Ökonomik ebenfalls erkannt. Allerdings werden auch in diesem Punkt die sich daraus ergebenden Konsequenzen nicht zu Ende gedacht. Denn Natur wird meist lediglich als Restriktion menschlichen Wirtschaftens konzipiert.

Diesem restriktiven Naturverständnis nach gilt es, die „Substanz“ der Natur zu "erhalten“. Es ist folglich die Rede von „ökologischen Leitplanken“, von „Tragekapazitäten“ und ähnlichen Konzepten, die scheinbar objektiv bestehen und le- diglich naturwissenschaftlich erkannt werden können.

Diese Metaphern werden weder der Tatsache gerecht, dass wir schon heute weitgehend von menschlich geprägter Natur umgeben sind, noch der Anforderung, die Leistungen von Natur fürs Wirtschaften konzeptionell $\mathrm{zu}$ fassen. Die Frage ist nicht allein, welche Grenzen der Naturbeanspruchung wir einhalten müssen, damit Ökosysteme nicht in ihrer bisherigen Funktionsweise zerstört werden, sondern wie wir Natur(produktivität) gestalten wollen. Welche handlungsleitenden Regeln lassen sich daraus ableiten?

\section{Große Fragen der Nachhaltigkeitsforschung}

Mit der Frage nach der wünschbaren Gestaltung von Natur werden in der feministischen Forschung neben dem normativen Prinzip der intergenerationellen Gerechtigkeit auch Fragen des guten Lebens angesprochen. Damit wirft sie große Fragen einer wohlverstandenen Nachhaltigkeitsforschung auf, die bisher auch von ihr selbst noch nicht hinreichend beantwortet worden sind.

Was macht den normativen Kern des Nachhaltigkeitsbegriffs aus und auf welche Weise sind darin Fragen der intergenerationellen Gerechtigkeit mit Fragen des guten Lebens verknüpft? Wo sind die Grenzen des Begriffs Nachhaltigkeit? Steht er Pate für eine in jeglicher Hinsicht wünschenswerte Entwicklung oder ist er nur ein Gesichtspunkt unter mehreren?

AUTORIN + KONTAKT

Dr. Tanja von Egan-Krieger ist promovierte Philosophin und Fellow am MeM - Denkfabrik für Wirtschaftsethik. Ihre Forschungsschwerpunkte sind Diskursethik und Kritische Theorie der Gesellschaft, Wirtschaftsethik, Nachhaltigkeit und intergenerationelle Gerechtigkeit, Politische Philosophie, Umweltethik und Feministische Philosophie.

MeM - Denkfabrik für Wirtschaftsethik, Wolframstraße 39, 12105 Berlin. E-Mail: tanja.egan@mem-wirtschaftsethik.de 